\title{
Periodic Solutions for a Prescribed Mean Curvature Equation with Multiple Delays
}

\author{
Shiping $L u^{1}$ and Ming $L u^{2}$ \\ ${ }^{1}$ College of Math and Statistics, Nanjing University of Information Science \& Technology, Nanjing 210044, China \\ ${ }^{2}$ Department of Mathematics, Anhui Normal University, Wuhu 241000, China
}

Correspondence should be addressed to Shiping Lu; lushiping88@sohu.com

Received 2 April 2014; Accepted 9 May 2014; Published 26 May 2014

Academic Editor: Meng Fan

Copyright (c) 2014 S. Lu and M. Lu. This is an open access article distributed under the Creative Commons Attribution License, which permits unrestricted use, distribution, and reproduction in any medium, provided the original work is properly cited.

We study the existence of periodic solutions for the one-dimensional prescribed mean curvature delay equation $(d / d t)\left(x^{\prime}(t) / \sqrt{1+\left(x^{\prime}(t)\right)^{2}}\right)+\sum_{i=1}^{n} a_{i}(t) g\left(x\left(t-\tau_{i}(t)\right)\right)=p(t)$. By using Mawhin's continuation theorem, a new result is obtained. Furthermore, the nonexistence of periodic solution for the equation is investigated as well.

\section{Introduction}

Prescribed mean curvature equation arises from some problems associated with differential geometry and physics such as combustible gas dynamics [1-3]. In the past years, the onedimensional mean curvature equation of autonomous type

$$
\frac{d}{d t}\left(\frac{x^{\prime}(t)}{\sqrt{1+\left(x^{\prime}(t)\right)^{2}}}\right)=g(x(t))
$$

has been studied by many authors [4-14]. The interesting thing is that most of them focus on the case in which the nonlinearity $g(u)$ is chosen to be various power growth functions. For example, Li and Liu in [4] studied the exact number of solutions for the boundary value problem

$$
\begin{gathered}
-\left(\frac{u^{\prime}}{\sqrt{1+\left(u^{\prime}\right)^{2}}}\right)^{\prime}=\lambda\left(u^{p}+u^{q}\right), \quad u>0 \text { in }(0,1), \\
u(0)=u(1)=0 .
\end{gathered}
$$

Clearly, the powers of growth with respect to the variable $u$ of functions on the right side of above two equations are not greater than $\max \{p, q\}$. Pan in [5] studied the exact multiple solutions of boundary value problem for a one-dimensional prescribed mean curvature equation with exponential nonlinearity

$$
\begin{array}{r}
\frac{d}{d t}\left(\frac{x^{\prime}(t)}{\sqrt{1+\left(x^{\prime}(t)\right)^{2}}}\right)=\lambda e^{x(t)}, \quad 0<t<L, \\
x(0)=x(L)=0 .
\end{array}
$$

Equation (3) can be viewed as a variant of the onedimensional Liouville-Bratu-Gelfand problem. By using the theory of time map, some results on the existence of multiple solutions are obtained. At the same time, we notice that Pan and Xing in [6] further studied the exact number of solutions for the problem

$$
\begin{array}{r}
\frac{d}{d t}\left(\frac{x^{\prime}(t)}{\sqrt{1+\left(x^{\prime}(t)\right)^{2}}}\right)=\lambda f(x(t)), \quad-L<t<L, \\
(-L)=x(L)=0,
\end{array}
$$

where $f(u)=e^{u}, f(u)=(1+u)^{p}$, and $f(u)=e^{u}-1$, respectively. For other recent developments and applications on the study of mean curvature equation, we refer the reader to [15-20], while the problem of periodic solution for prescribed mean curvature equation has been rarely 
studied [21-24]. Considering the delay phenomenon which exists generally in nature, Feng [22] studied the existence of periodic solutions for the one-dimensional mean curvature type equation in the following form:

$$
\begin{gathered}
\frac{d}{d t}\left(\frac{x^{\prime}(t)}{\sqrt{1+\left(x^{\prime}(t)\right)^{2}}}\right)+f(x(t)) x^{\prime}(t) \\
+g(t, x(t-\tau(t)))=p(t) .
\end{gathered}
$$

By imposing some conditions on functions $f$ and $g$ as follows.

(H) there are two constants $l>0$ and $\gamma>0$ such that

$$
\begin{gathered}
\left|g\left(t, x_{1}\right)-g\left(t, x_{2}\right)\right| \leq l\left|x_{1}-x_{2}\right| \quad \forall(t, x) \in R^{2} ; \\
|f(x)| \geq \gamma \quad \forall x \in R ;
\end{gathered}
$$

the author obtained that (3) has at least one periodic solution by using Mawhin's continuation theorem. From [22], we see that assumption $(\mathrm{H})$ is crucial for estimating a priori bounds of all possible $T$-periodic solutions.

In this paper, we consider the following prescribed mean curvature equation with multiple delays:

$$
\frac{d}{d t}\left(\frac{x^{\prime}(t)}{\sqrt{1+\left(x^{\prime}(t)\right)^{2}}}\right)+\sum_{i=1}^{n} a_{i}(t) g\left(x\left(t-\tau_{i}(t)\right)\right)=p(t)
$$

where $g \in C(R, R)$ and $p, a_{i}$, and $\tau_{i}$ are all continuous $T$-periodic functions, $i=1,2, \ldots, n$. By using Mawhin's continuation theorem, some new results are obtained; and the problem of nonexistence of periodic solution for (8) is investigated as well.

The significance of this paper lies in the following two respects: firstly, we do not need assumption (7); secondly, the conditions imposed on function $g(u)$ and the methods to estimate a priori bounds of possible $T$-periodic solutions for the equation $L x=\lambda N x$ are all essentially different from corresponding ones of [22]. For example, we do not require that the function $g$ satisfies global Lipschitz condition (6). Especially, the function $g$ is allowed to be exponential nonlinearity.

\section{Preliminaries}

In order to investigate the existence of periodic solutions for (8), we give some definitions and lemmas in this section.

In this paper, unless otherwise specified, we use the following notation. Let $P_{T}=\{\varphi \in C(R, R): \varphi(t+T) \equiv \varphi(t)\}$ with the norm defined by $|\varphi|_{\infty}=\max _{t \in[0, T]}|\varphi(t)| ; C_{T}=\{x=$ $\left.\left(x_{1}, x_{2}\right)^{\mathrm{T}}: x_{i} \in C(R, R), x_{i}(t) \equiv x_{i}(t+T), i=1,2\right\}$ and the norm defined by $\|x\|=\max \left\{\left|x_{1}\right|_{\infty},\left|x_{2}\right|_{\infty}\right\}$. Clearly, $P_{T}$ and $C_{T}$ are two Banach spaces. Furthermore, define $|\varphi|_{p}=$ $\left(\int_{0}^{T}|\varphi(s)|^{p} d s\right)^{1 / p}$ for all $\varphi \in P_{T}$, where $p>1$ is a constant.

Lemma 1 (see [25]). Suppose $\tau \in P_{T}$ and $\tau^{\prime}(t)<1, \forall t \in$ $[0, T]$. Then the function $t-\tau(t)$ has its inverse $\mu(t)$ satisfying $\mu \in C(R, R)$ with $\mu(t+T) \equiv \mu(t)+T, \forall t \in R$. Furthermore, if $\alpha, \beta \in P_{T}$, then

$$
\begin{gathered}
\int_{0}^{T} \alpha(t) \beta(t-\tau(t)) d t=\int_{0}^{T} \frac{\alpha(\mu(t))}{1-\tau^{\prime}(\mu(t))} \beta(t) d t \\
\int_{0}^{T} \frac{\alpha(\mu(t))}{1-\tau^{\prime}(\mu(t))} d t=\int_{0}^{T} \alpha(t) d t
\end{gathered}
$$

Now, let us recall Mawhin's continuation theorem. Let $X$ and $Y$ be real Banach spaces and let $L: D(L) \subset X \rightarrow Y$ be a Fredholm operator with index zero; here $D(L)$ denotes the domain of $L$. This means that $\operatorname{Im} L$ is closed in $Y$ and $\operatorname{dim} \operatorname{ker} L=\operatorname{codim} \operatorname{Im} L<+\infty$. If $L$ is a Fredholm operator with index zero, then there exist continuous projectors $P$ : $X \rightarrow X, Q: Y \rightarrow Y$ such that $\operatorname{Im} P=\operatorname{ker} L, \operatorname{Im} L=\operatorname{ker} Q$ and $L_{D(L) \cap \text { ker } P}:(I-P) X \rightarrow \operatorname{Im} L$ is invertible. Denote by $K_{p}$ the inverse of $L_{P}$.

Let $\Omega$ be an open bounded subset of $X$; a continuous map $N: \bar{\Omega} \subset X \rightarrow Y$ is said to be $L$-compact in $\bar{\Omega}$ if $Q N(\bar{\Omega})$ is bounded and the operator $K_{p}(I-Q) N(\bar{\Omega})$ is relatively compact.

Lemma 2 (see [26]). Suppose that $X$ and $Y$ are two Banach spaces, and $L: D(L) \subset X \rightarrow Y$ is a Fredholm operator with index zero. Furthermore, $\Omega \subset X$ is an open bounded set and $N: \bar{\Omega} \rightarrow Y$ is L-compact on $\bar{\Omega}$. If all the following conditions hold:

(1) $L x \neq \lambda N x, \forall x \in \partial \Omega \cap D(L), \lambda \in(0,1)$,

(2) $N x \notin \operatorname{Im} L, \forall x \in \partial \Omega \cap \operatorname{ker} L$,

(3) $\operatorname{deg}\{J Q N, \Omega \cap \operatorname{ker} L, 0\} \neq 0$, where $J: \operatorname{Im} Q \rightarrow \operatorname{ker} L$ is an isomorphism,

then equation $L x=N x$ has a solution on $\bar{\Omega} \cap D(L)$.

Throughout this paper, for each $i \in\{1,2, \ldots, n\}$, besides $\tau_{i} \in P_{T}$, we suppose in addition $\tau_{i} \in C^{1}(R, R)$ with $\tau^{\prime}(t)<1$, $\forall t \in[0, T]$.

Remark 3. From above assumption, one can find from Lemma 1 that, for each $i \in\{1,2, \ldots, n\}$, the function $t-\tau_{i}(t)$ has its inverse denoted by $\mu(t)$. Define

$$
\begin{gathered}
\Gamma(t):=\sum_{i=1}^{n} \frac{a_{i}\left(\mu_{i}(t)\right)}{1-\tau_{i}^{\prime}\left(\mu_{i}(t)\right)}, \\
\Gamma_{1}(t):=\sum_{i=1}^{n}\left|\frac{a_{i}\left(\mu_{i}(t)\right)}{1-\tau_{i}^{\prime}\left(\mu_{i}(t)\right)}\right| .
\end{gathered}
$$

Since $a_{i} \in P_{T}$, it follows from Lemma 1 again that

$$
\int_{0}^{T} \Gamma(t) d t=\sum_{i=1}^{n} \int_{0}^{T} a_{i}(t) d t, \quad \int_{0}^{T} \Gamma_{1}(t) d t=\sum_{i=1}^{n} \int_{0}^{T}\left|a_{i}(t)\right| d t .
$$

For the sake of convenience, we list the following assumptions which will be used for us to study the existence of periodic solutions to (8) in Section 3. 
(A1) The functions $\Gamma, \Gamma_{1}$, and $p$ satisfy $\Gamma(t)>0$ for all $t \in[0, T]$ and

$$
\sigma:=\left|\frac{\Gamma_{1}}{\Gamma}\right|_{\infty} \int_{0}^{T} p(s) d s+\int_{0}^{T}|p(s)| d s \in(0,1),
$$

where $\Gamma$ and $\Gamma_{1}$ are all continuous functions determined by Remark 3.

(A2) The function $g$ satisfies $g(x)>0$ for all $x \in R$, and there is a constant $M>0$ such that, for all $x \in(M,+\infty)$,

$$
\begin{aligned}
& -g(x)\left(\sum_{i=1}^{n} \int_{0}^{T} a_{i}(s) d s\right)+\int_{0}^{T} p(s) d s<0 \\
& -g(-x)\left(\sum_{i=1}^{n} \int_{0}^{T} a_{i}(s) d s\right)+\int_{0}^{T} p(s) d s>0 .
\end{aligned}
$$

Remark 4. Since (8) does not contain the term $f(x(t)) x^{\prime}(t)$, condition (7) in assumption (H) of [22] does not hold. Furthermore, in our paper, the function $g$ is not required to satisfy the global Lipschitz condition (6). So the conditions in our paper are all essentially different from corresponding ones of [22].

Since the differential term of $x^{\prime}(t) / \sqrt{1+\left(x^{\prime}(t)\right)^{2}}$ is nonlinear with respect to $x(t)$, the differential operator $L:(L x)(t)=(d / d t)\left(x^{\prime}(t) / \sqrt{1+\left(x^{\prime}(t)\right)^{2}}\right)$ associated with Mawhin's continuation Theorem is not Fredholm type. So we need to convert (8) to the following two-dimensional system:

$$
\begin{gathered}
x_{1}^{\prime}(t)=\frac{x_{2}(t)}{\sqrt{1-\left(x_{2}(t)\right)^{2}}}, \\
x_{2}^{\prime}(t)=-\sum_{i=1}^{n} a_{i}(t) g\left(x_{1}\left(t-\tau_{i}(t)\right)\right)+p(t) .
\end{gathered}
$$

Clearly, if $\left(x_{1}(t), x_{2}(t)\right)^{\mathrm{T}}$ is a T-periodic solution to (15), then $x_{1}(t)$ must be a $T$-periodic solution to (8). From this, we see that, in order to investigate the existence of $T$-periodic solution for (8), it suffices for us to prove that (15) has a $T$ periodic solution.

For using Mawhin's continuation theorem, let

$$
\begin{gathered}
L: D(L) \longrightarrow C_{T}, \quad[L x](t)=\left(x_{1}^{\prime}(t), x_{2}^{\prime}(t)\right)^{\mathrm{T}}, \quad t \in R, \\
N: \Sigma \subset C_{T} \longrightarrow C_{T}, \\
{[N x](t)=\left(\begin{array}{c}
\psi\left(x_{2}(t)\right) \\
\left.-\sum_{i=1}^{n} a_{i}(t) g\left(x_{1}\left(t-\tau_{i}(t)\right)\right)+p(t)\right), \quad t \in R,
\end{array}\right.}
\end{gathered}
$$

where $\Sigma=\left\{x: x=\left(x_{1}, x_{2}\right)^{\mathrm{T}} \in C_{T}\right.$ with $\left|x_{2}\right|_{\infty}<$ $\left.\delta,\left|x_{1}\right|_{\infty}<+\infty\right\}$, where $\delta \in(\sigma, 1)$ is a constant and $\sigma \in(0,1)$ is determined in assumption (A1).

\section{Main Results}

In this section, we will apply Lemma 2 to study the existence of periodic solutions for (8).
Theorem 5. Suppose that assumptions (A1) and (A2) hold. Then (8) possesses at least one T-periodic solution.

Proof. Suppose that $u \in C_{T}$ is an arbitrary solution to the equation $L x=\lambda N x$ for each $\lambda \in(0,1)$, where $L$ and $N$ are defined by (16), respectively. This implies

$$
\begin{gathered}
u_{1}^{\prime}(t)=\frac{\lambda u_{2}(t)}{\sqrt{1-\left(u_{2}(t)\right)^{2}}}, \\
u_{2}^{\prime}(t)=-\lambda \sum_{i=1}^{n} a_{i}(t) g\left(u_{1}\left(t-\tau_{i}(t)\right)\right)+\lambda p(t), \\
\lambda \in(0,1) .
\end{gathered}
$$

From the first formula of (18), we see

$$
u_{2}(t)=\frac{(1 / \lambda) u_{1}^{\prime}(t)}{\sqrt{1+\left(u_{2}^{\prime}(t)\right)^{2} / \lambda^{2}}}, \quad \lambda \in(0,1) .
$$

Substituting (18) into the second formula of (17), we have

$$
\begin{aligned}
& \frac{d}{d t}\left(\frac{(1 / \lambda) u_{1}^{\prime}(t)}{\sqrt{1+\left(u_{1}^{\prime}(t)\right)^{2} / \lambda^{2}}}\right) \\
& \quad=-\lambda \sum_{i=1}^{n} a_{i}(t) g\left(u_{1}\left(t-\tau_{i}(t)\right)\right)+\lambda p(t) .
\end{aligned}
$$

Integrating both sides of (19) on the interval $[0, T]$, we obtain

$$
\int_{0}^{T} \sum_{i=1}^{n} a_{i}(t) g\left(u_{1}\left(t-\tau_{i}(t)\right)\right) d t=\int_{0}^{T} p(t) d t .
$$

Since $\tau_{i}^{\prime}(t)<1$ for all $t \in[0, T]$, by using Lemma 1 , we see that the function $t-\tau_{i}(t)$ has its inverse $\mu_{i}(t)(i=1,2, \ldots, n)$. So by applying (9) to (20), we have

$$
\int_{0}^{T} \Gamma(t) g\left(u_{1}(t)\right) d t=\int_{0}^{T} p(t) d t
$$

where $\Gamma(t)=\sum_{i=1}^{n}\left(a_{i}\left(\mu_{i}(t)\right) /\left(1-\tau_{i}^{\prime}\left(\mu_{i}(t)\right)\right)\right)$ is determined by Remark 3. Similarly, from (19), we have

$$
\begin{aligned}
& \int_{0}^{T}\left|\left(\frac{(1 / \lambda) u_{1}^{\prime}(t)}{\sqrt{1+\left(u_{1}^{\prime}(t)\right)^{2} / \lambda^{2}}}\right)^{\prime}\right| d t \\
& \quad \leq \lambda \int_{0}^{T} \Gamma_{1}(t)\left|g\left(u_{1}(t)\right)\right| d t+\lambda \int_{0}^{T}|p(t)| d t,
\end{aligned}
$$

which together with the condition of $g(x)>0$ for all $x \in R$ in assumption (A2) yields that

$$
\begin{aligned}
& \int_{0}^{T}\left|\left(\frac{(1 / \lambda) u_{1}^{\prime}(t)}{\sqrt{1+\left(u_{1}^{\prime}(t)\right)^{2} / \lambda^{2}}}\right)^{\prime}\right| d t \\
& \quad \leq \int_{0}^{T} \Gamma_{1}(t) g\left(u_{1}(t)\right) d t+\int_{0}^{T}|p(t)| d t .
\end{aligned}
$$


It follows from the condition of $\Gamma(t)>0$ for all $t \in[0, T]$ in assumption (A1) that

$$
\begin{aligned}
& \int_{0}^{T}\left|\left(\frac{(1 / \lambda) u_{1}^{\prime}(t)}{\sqrt{1+\left(u_{1}^{\prime}(t)\right)^{2} / \lambda^{2}}}\right)^{\prime}\right| d t \\
& \leq \int_{0}^{T} \frac{\Gamma_{1}(t)}{\Gamma(t)} \Gamma(t) g\left(u_{1}(t)\right) d t+\int_{0}^{T}|p(t)| d t \\
& \leq\left|\frac{\Gamma_{1}}{\Gamma}\right|_{\infty} \int_{0}^{T} \Gamma(t) g\left(u_{1}(t)\right) d t+\int_{0}^{T}|p(t)| d t .
\end{aligned}
$$

Substituting (21) into the above formula, we obtain that

$$
\begin{aligned}
& \int_{0}^{T}\left|\left(\frac{(1 / \lambda) u_{1}^{\prime}(t)}{\sqrt{1+\left(u_{1}^{\prime}(t)\right)^{2} / \lambda^{2}}}\right)^{\prime}\right| d t \\
& \quad \leq\left|\frac{\Gamma_{1}}{\Gamma}\right|_{\infty} \int_{0}^{T} p(t) d t+\int_{0}^{T}|p(t)| d t .
\end{aligned}
$$

On the other hand, since $\int_{0}^{T} u_{1}^{\prime}(s) d s=u_{1}(T)-u_{1}(0)=0$, there must be a point $t^{*} \in[0, T]$ such that $u_{1}^{\prime}\left(t^{*}\right)=0$; that is, $(1 / \lambda) u_{1}^{\prime}\left(t^{*}\right) / \sqrt{1+\left(u_{1}^{\prime}\left(t^{*}\right)\right)^{2} / \lambda^{2}}=0$. So

$$
\begin{aligned}
&\left|\frac{(1 / \lambda) u_{1}^{\prime}(t)}{\sqrt{1+\left(u_{1}^{\prime}(t)\right)^{2} / \lambda^{2}}}\right| \leq \int_{t^{*}}^{t}\left|\left(\frac{(1 / \lambda) u_{1}^{\prime}(s)}{\sqrt{1+\left(u_{1}^{\prime}(s)\right)^{2} / \lambda^{2}}}\right)^{\prime}\right| d s \\
& \leq \int_{t^{*}}^{t^{*}+T}\left|\left(\frac{(1 / \lambda) u_{1}^{\prime}(s)}{\sqrt{1+\left(u_{1}^{\prime}(s)\right)^{2} / \lambda^{2}}}\right)^{\prime}\right| d s \\
&=\int_{0}^{T}\left|\left(\frac{(1 / \lambda) u_{1}^{\prime}(s)}{\sqrt{1+\left(u_{1}^{\prime}(s)\right)^{2} / \lambda^{2}}}\right)^{\prime}\right| d s, \\
& t \in\left[t^{*}, t^{*}+T\right] .
\end{aligned}
$$

This together with (25) implies that

$$
\begin{array}{r}
\left|\frac{(1 / \lambda) u_{1}^{\prime}(t)}{\sqrt{1+\left(u_{1}^{\prime}(t)\right)^{2} / \lambda^{2}}}\right| \leq\left|\frac{\Gamma_{1}}{\Gamma}\right|_{\infty} \int_{0}^{T} p(t) d t+\int_{0}^{T}|p(t)| d t \\
t \in\left[t^{*}, t^{*}+T\right] .
\end{array}
$$

From assumption (A1), we know $\sigma=\left|\Gamma_{1} / \Gamma\right|_{\infty} \int_{0}^{T} p(t) d t+$ $\int_{0}^{T}|p(t)| d t<1$; it follows from (18) and (27) that

$$
\left|u_{2}\right|_{\infty}=\max _{t \in\left[t^{*}, t^{*}+T\right]}\left|\frac{(1 / \lambda) u_{1}^{\prime}(t)}{\sqrt{1+\left(u_{1}^{\prime}(t)\right)^{2} / \lambda^{2}}}\right| \leq \sigma<1 .
$$

Furthermore, from the fact of the function $x / \sqrt{1+x^{2}}$ being strongly increasing for $x \in R$, it follows from (27) that

$$
\left|\frac{u_{1}^{\prime}(t)}{\lambda}\right| \leq \frac{\sigma}{\sqrt{1-\sigma^{2}}}, \quad t \in\left[t^{*}, t^{*}+T\right] ;
$$

that is,

$$
\left|u_{1}^{\prime}\right|_{\infty}=\max _{t \in\left[t^{*}, t^{*}+T\right]}\left|u_{1}^{\prime}(t)\right| \leq \frac{\lambda \sigma}{\sqrt{1-\sigma^{2}}}<\frac{\sigma}{\sqrt{1-\sigma^{2}}}:=M_{0} .
$$

By using (28), we have

$$
\begin{aligned}
\frac{1}{\sqrt{1+M_{0}^{2}}} \int_{0}^{T}\left|u_{1}^{\prime}(t)\right| d t & =\frac{1}{\sqrt{1+M_{0}^{2}}} \int_{t^{*}}^{t^{*}+T}\left|u_{1}^{\prime}(t)\right| d t \\
& <\int_{t^{*}}^{t^{*}+T}\left|\frac{u_{1}^{\prime}(t)}{\sqrt{\lambda^{2}+\left(u_{1}^{\prime}(t)\right)^{2}}}\right| d t \\
& =\int_{t^{*}}^{t^{*}+T}\left|\frac{(1 / \lambda) u_{1}^{\prime}(t)}{\sqrt{1+\left(u_{1}^{\prime}(t)\right)^{2} / \lambda^{2}}}\right| d t \\
& \leq T \sigma,
\end{aligned}
$$

which implies

$$
\int_{0}^{T}\left|u_{1}^{\prime}(t)\right| d t<T \sigma \sqrt{1+M_{0}^{2}}
$$

Furthermore, from (21) and the condition of $\Gamma(t)>0$ for all $t \in[0, T]$ in assumption (A1), we see that there must be a point $t_{1} \in[0, T]$ such that

$$
g\left(u_{1}\left(t_{1}\right)\right) \int_{0}^{T} \Gamma(s) d s=\int_{0}^{T} p(s) d s
$$

that is,

$$
g\left(u_{1}\left(t_{1}\right)\right)=\frac{\int_{0}^{T} p(s) d s}{\int_{0}^{T} \Gamma(s) d s} .
$$

By using the conclusion of $\int_{0}^{T} \Gamma(s) d s=\sum_{i=1}^{n} \int_{0}^{T} a_{i}(s) d s$ in Remark 3, we have

$$
g\left(u_{1}\left(t_{1}\right)\right)=\frac{\int_{0}^{T} p(s) d s}{\sum_{i=1}^{n} \int_{0}^{T} a_{i}(s) d s} .
$$

So by using assumption (A2), we see

$$
\left|u_{1}\left(t_{1}\right)\right| \leq M,
$$

which together with (32) yields that

$$
\begin{aligned}
\left|u_{1}\right|_{\infty} & =\max _{t \in\left[t_{1}, t_{1}+T\right]}\left|u_{1}(t)\right| \leq\left|u_{1}\left(t_{1}\right)\right|+\int_{t_{1}}^{t_{1}+T}\left|u_{1}^{\prime}(s)\right| d s \\
& \leq M+\int_{0}^{T}\left|u_{1}^{\prime}(t)\right| d t<M+T \sigma \sqrt{1+M_{0}^{2}} \\
& =: M_{1} .
\end{aligned}
$$


If set $\Omega=\left\{x \in C_{T}:\left|x_{1}\right|_{\infty}<M_{1},\left|x_{2}\right|_{\infty}<\sigma\right\}$, then, from the above proof, we see that $L u \neq \lambda N u$ for all $(\lambda, u) \in(0,1) \times \partial \Omega$. This means that condition (1) of Lemma 2 holds.

Now, suppose $u \in \partial \Omega \cap \operatorname{ker} L$; then $u=\left(u_{1}, u_{2}\right) \in R^{2}$ is a constant vector with $\left|u_{1}\right|=M_{1}$ or $\left|u_{2}\right|=\sigma$. So

$$
Q N u=\left\{\begin{array}{l}
\psi\left(u_{2}\right)=\frac{u_{2}}{\sqrt{1-u_{2}^{2}}} \\
-g\left(u_{1}\right)\left(\sum_{i=1}^{n} \int_{0}^{T} a_{i}(s) d s\right)+\int_{0}^{T} p(s) d s
\end{array}\right.
$$

which together with assumption (A2) yields that

$$
Q N u \neq 0, \quad \forall u \in \partial(\Omega \cap \operatorname{ker} L) .
$$

From (30), (37), and (39), we see that conditions (1) and (2) in Lemma 2 hold for $\Omega=\left\{x \in C_{T}:\left\|x_{1}\right\|_{\infty}<M_{1},\left\|x_{2}\right\|_{\infty}<\sigma\right\}$.

Below, we will show that condition (3) of Lemma 2 also holds. In fact, let

$$
H(\mu, x)=\mu x+(1-\mu) J Q N x, \quad \text { for }(\mu, x) \in[0,1] \times \bar{\Omega},
$$

where $J\left(x_{1}, x_{2}\right)^{\mathrm{T}}=\left(x_{1}, x_{2}\right)^{\mathrm{T}}$. Clearly, if $u^{*}(t)=\left(u_{1}^{*}(t)\right.$, $\left.u_{2}^{*}(t)\right)^{\mathrm{T}} \in \bar{\Omega} \cap \operatorname{ker} L$ is the solution of equation $H(\mu, x)=0$ for some $\mu \in[0,1]$, then $\left(u_{1}^{*}(t), u_{2}^{*}(t)\right)^{\mathrm{T}}$ is a constant vector. So

$$
\begin{gathered}
\psi\left(u_{2}^{*}\right)=\frac{u_{2}^{*}}{\sqrt{1-\left(u_{2}^{*}\right)^{2}}}=0 \\
-g\left(u_{1}^{*}\right)\left(\sum_{i=1}^{n} \int_{0}^{T} a_{i}(s) d s\right)+\int_{0}^{T} p(s) d s=0 .
\end{gathered}
$$

This together with assumption (A2) results in $u_{2}^{*}=0$ and $\left|u_{1}^{*}\right| \leq M$. By (37), we see that $M<M_{1}$, and then $u^{*} \notin$ $\partial(\bar{\Omega} \cap \operatorname{ker} L)$. From this, we conclude that $H(\mu, x) \neq 0$ for all $(\mu, x) \in[0,1] \times \partial(\bar{\Omega} \cap \operatorname{ker} L)$, which together with the property of homotopy invariance for Brouwer's topological degree gives that

$$
\begin{aligned}
\operatorname{deg}\{J Q N, \Omega \cap \operatorname{ker} L, 0\} & =\operatorname{deg}\{H(0, \cdot), \Omega \cap \operatorname{ker} L, 0\} \\
& =\operatorname{deg}\{H(1, \cdot), \Omega \cap \operatorname{ker} L, 0\} \\
& =\operatorname{deg}\{I, \Omega \cap \operatorname{ker} L, 0\} \\
& =1 \neq 0 .
\end{aligned}
$$

This proves that condition (3) of Lemma 2 holds. Thus, by using Lemma 2, we have that (15) possesses at least one $T$ periodic solution $u_{0}(t)=\left(u_{01}(t), u_{02}(t)\right)^{\mathrm{T}} \in \bar{\Omega}=\left\{x \in C_{T}\right.$ : $\left.\left|x_{1}\right|_{\infty} \leq M_{1},\left|x_{2}\right|_{\infty} \leq \sigma\right\}$. Clearly, $u_{01}(t)$ must be a $T$-periodic solution to (8).

Remark 6. From the proof of Theorem 5, it is easy to see that assumption (A2) can be replaced by the following:
$(\mathrm{A} 2)^{\prime}$ the function $g$ satisfies $g(x)>0$ for all $x \in R$, and there is a constant $M>0$ such that, for all $x \in$ $(M,+\infty)$,

$$
\begin{aligned}
& -g(x)\left(\sum_{i=1}^{n} \int_{0}^{T} a_{i}(s) d s\right)+\int_{0}^{T} p(s) d s>0, \\
& -g(-x)\left(\sum_{i=1}^{n} \int_{0}^{T} a_{i}(s) d s\right)+\int_{0}^{T} p(s) d s<0 .
\end{aligned}
$$

Theorem 7. Suppose that $\Gamma(t) \geq 0$ and $\Gamma(t) \not \equiv$ for $t \in[0, T]$. If

$$
\frac{\int_{0}^{T} p(s) d s}{\sum_{i=1}^{n} \int_{0}^{T} a_{i}(s) d s} \notin g(R):=\{g(u): u \in R\},
$$

then (8) has no T-periodic solution.

Suppose that $u_{0}(t)$ is a $T$-periodic solution to (8). Then

$$
\frac{d}{d t}\left(\frac{u_{0}^{\prime}(t)}{\sqrt{1+\left(u_{0}^{\prime}(t)\right)^{2}}}\right)+\sum_{i=1}^{n} a_{i}(t) g\left(u_{0}\left(t-\tau_{i}(t)\right)\right)=p(t) \text {. }
$$

Integrating both sides of $(45)$ on $[0, T]$, we have

$$
\int_{0}^{T} \Gamma(s) g\left(u_{0}(s)\right) d s=\int_{0}^{T} p(s) d s .
$$

Since $\Gamma(t) \geq 0$ and $\Gamma(t) \neq 0$ for $t \in[0, T]$, it follows that there is a $t_{0} \in[0, T]$ such that $g\left(u_{0}\left(t_{0}\right)\right)=\int_{0}^{T} p(s) d s / \int_{0}^{T} \Gamma(s) d s$. In view of the fact of $\int_{0}^{T} \Gamma(s) d s=\sum_{i=1}^{n} \int_{0}^{T} a_{i}(s) d s$ in Remark 3 , we see that

$$
g\left(u_{0}\left(t_{0}\right)\right)=\frac{\int_{0}^{T} p(s) d s}{\sum_{i=1}^{n} \int_{0}^{T} a_{i}(s) d s} .
$$

So

$$
\frac{\int_{0}^{T} p(s) d s}{\sum_{i=1}^{n} \int_{0}^{T} a_{i}(s) d s} \in g(R),
$$

which contradicts the assumption of $\int_{0}^{T} p(s) d s / \sum_{i=1}^{n} \int_{0}^{T} a_{i}(s) d s$ $\notin g(R)$.

For illustrating Theorem 5, we give the following examples.

Example 8. Consider the problem of existence of periodic solution to the equation

$$
\begin{aligned}
& \left(\frac{x^{\prime}(t)}{\sqrt{1+\left(x^{\prime}(t)\right)^{2}}}\right)^{\prime}+\frac{1}{4}\left(1-\frac{1}{2} \cos t\right) e^{x(t-(1 / 2) \sin t)} \\
& \quad-\frac{1}{8}\left(1+\frac{1}{2} \sin t\right) e^{x(t-(1 / 2) \cos t)} \\
& =\frac{1}{5 \pi} \sin ^{2} t
\end{aligned}
$$


Corresponding to (8), we see that $g(x)=e^{x}, a_{1}(t)=(1 / 4)(1-$ $(1 / 2) \cos t), a_{2}(t)=-(1 / 8)(1+(1 / 2) \sin t), \tau_{1}(t)=(1 / 2) \sin t$, $\tau_{2}(t)=(1 / 2) \cos t$, and $p(t)=(1 / 5 \pi) \sin ^{2} t$. Let $\mu_{i}(t)$ be the inverse of $t-\tau_{i}(t),(i=1,2)$. Then

$$
\begin{aligned}
\Gamma(t) & =\frac{a_{1}\left(\mu_{1}(t)\right)}{1-\tau_{1}^{\prime}\left(\mu_{1}(t)\right)}+\frac{a_{2}\left(\mu_{2}(t)\right)}{1-\tau_{2}^{\prime}\left(\mu_{2}(t)\right)} \\
& =\frac{1-(1 / 2) \cos \mu_{1}(t)}{4\left(1-(1 / 2) \cos \mu_{1}(t)\right)}-\frac{1+(1 / 2) \sin \mu_{2}(t)}{8\left(1+(1 / 2) \sin \mu_{2}(t)\right)} \\
& =\frac{1}{8}>0, \quad \forall t \in[0,2 \pi],
\end{aligned}
$$

and $\left|\Gamma_{1} / \Gamma\right|_{\infty}=3$. So

$$
\begin{aligned}
\sigma & :=\left|\frac{\Gamma_{1}}{\Gamma}\right|_{\infty} \int_{0}^{T} p(s) d s+\int_{0}^{T}|p(s)| d s \\
& =\frac{4}{5 \pi} \int_{0}^{2 \pi} \sin ^{2} s d s=\frac{4}{5}<1,
\end{aligned}
$$

which implies that assumption (A1) holds. Furthermore, since $g(x)=e^{x}$, assumption (A2) also holds. Thus, by using Theorem 5 , we have that (49) possesses at least one $2 \pi$ periodic solution.

Remark 9. Since $g(x)=e^{x}$, it is easy to see that the function $g$ does not satisfy the global Lipschitz condition (6). So the result of Example 8 cannot be obtained by using the main theorem of [22].

Example 10. Consider the problem of existence of periodic solution to the equation

$$
\begin{aligned}
& \left(\frac{x^{\prime}(t)}{\sqrt{1+\left(x^{\prime}(t)\right)^{2}}}\right)^{\prime}+\frac{1}{4}\left(1-\frac{1}{2} \cos t\right) \frac{e^{x(t-(1 / 2) \sin t)}}{1+e^{x(t-(1 / 2) \sin t)}} \\
& \quad+\frac{1}{8}\left(1+\frac{1}{2} \sin t\right) \frac{e^{x(t-(1 / 2) \cos t)}}{1+e^{x(t-(1 / 2) \cos t)}} \\
& =\theta \sin ^{2} t,
\end{aligned}
$$

where $\theta \in R$ is a constant. Corresponding to (8), we have

$$
\begin{gathered}
\Gamma(t) \equiv \frac{3}{8}, \quad\left|\frac{\Gamma_{1}}{\Gamma}\right|_{\infty}=1, \\
g(u)=\frac{e^{u}}{1+e^{u}}, \quad \frac{\int_{0}^{2 \pi} p(s) d s}{\sum_{i=1}^{n} \int_{0}^{2 \pi} a_{i}(s) d s}=\frac{4}{3} \theta .
\end{gathered}
$$

Thus, if $\theta \in(0,3 / 4)$, then there must be a constant $M>0$ such that

$$
\begin{aligned}
& -\frac{e^{x}}{1+e^{x}} \int_{0}^{2 \pi} \Gamma(s) d s+\theta \int_{0}^{2 \pi} \sin ^{2} s d s \\
& =-\frac{3 \pi e^{x}}{4\left(1+e^{x}\right)}+\theta \pi \\
& <0, \\
& -\frac{e^{-x}}{1+e^{-x}} \int_{0}^{2 \pi} \Gamma(s) d s+\theta \int_{0}^{2 \pi} \sin ^{2} s d s \\
& =-\frac{3 \pi e^{-x}}{4\left(1+e^{-x}\right)}+\theta \pi \\
& >0
\end{aligned}
$$

for $x>M$, and if $\theta \in(0,1 / 2 \pi)$, then

$$
\begin{aligned}
\sigma & :=\left|\frac{\Gamma_{1}}{\Gamma}\right|_{\infty} \int_{0}^{T} p(s) d s+\int_{0}^{T}|p(s)| d s \\
& =2 \theta \int_{0}^{2 \pi} \sin ^{2} s d s=2 \theta \pi \\
& <1 .
\end{aligned}
$$

Furthermore, since $g(R)=(0,1)$, it follows that $\int_{0}^{2 \pi} p(s) d s /$ $\sum_{i=1}^{n} \int_{0}^{2 \pi} a_{i}(s) d s=(4 / 3) \theta \notin g(R)$ for $\theta \in(-\infty, 0] \cup[3 / 4,+\infty)$. Therefore, by using Theorems 5 and 7 , we obtain the following results:

(1) if $\theta \in(0,1 / 2 \pi)$, then (52) has at least one $2 \pi$-periodic solution;

(2) if $\theta \in(-\infty, 0] \cup[3 / 4,+\infty)$, (52) has no $2 \pi$-periodic solution.

\section{Conflict of Interests}

The authors declare that there is no conflict of interests regarding the publication of this paper.

\section{Acknowledgments}

The authors are very grateful to the referee for her/his careful reading of the original paper and for her/his valuable suggestions for improving this paper. The authors thank the referees for valuable comments and suggestions. This work was sponsored by the NSFC 11271197 of China.

\section{References}

[1] M. Bergner, "On the Dirichlet problem for the prescribed mean curvature equation over general domains," Differential Geometry and Its Applications, vol. 27, no. 3, pp. 335-343, 2009.

[2] O. Rey, "Heat flow for the equation of surfaces with prescribed mean curvature," Mathematische Annalen, vol. 291, no. 1, pp. 123-146, 1991. 
[3] P. Amster and M. C. Mariani, "The prescribed mean curvature equation for nonparametric surfaces," Nonlinear Analysis. Theory, Methods \& Applications, vol. 52, no. 4, pp. 1069-1077, 2003.

[4] W. Li and Z. Liu, "Exact number of solutions of a prescribed mean curvature equation," Journal of Mathematical Analysis and Applications, vol. 367, no. 2, pp. 486-498, 2010.

[5] H. Pan, "One-dimensional prescribed mean curvature equation with exponential nonlinearity," Nonlinear Analysis. Theory, Methods \& Applications, vol. 70, no. 2, pp. 999-1010, 2009.

[6] H. Pan and R. Xing, "Time maps and exact multiplicity results for one-dimensional prescribed mean curvature equations. II," Nonlinear Analysis. Theory, Methods \& Applications, vol. 74, no. 11, pp. 3751-3768, 2011.

[7] P. Amster and M. C. Mariani, "The prescribed mean curvature equation for nonparametric surfaces," Nonlinear Analysis. Theory, Methods \& Applications, vol. 52, no. 4, pp. 1069-1077, 2003.

[8] P. Benevieri, J. M. do Ó, and E. S. de Medeiros, "Periodic solutions for nonlinear systems with mean curvature-like operators," Nonlinear Analysis. Theory, Methods \& Applications, vol. 65, no. 7, pp. 1462-1475, 2006.

[9] D. Bonheure, P. Habets, F. Obersnel, and P. Omari, "Classical and non-classical positive solutions of a prescribed curvature equation with singularities," Rendiconti dell'Instituto di Matematica dell'Università di Trieste, vol. 39, pp. 63-85, 2007.

[10] D. Bonheure, P. Habets, F. Obersnel, and P. Omari, "Classical and non-classical solutions of a prescribed curvature equation," Journal of Differential Equations, vol. 243, no. 2, pp. 208-237, 2007.

[11] P. Habets and P. Omari, "Positive solutions of an indefinite prescribed mean curvature problem on a general domain," Advanced Nonlinear Studies, vol. 4, no. 1, pp. 1-13, 2004.

[12] P. Habets and P. Omari, "Multiple positive solutions of a onedimensional prescribed mean curvature problem," Communications in Contemporary Mathematics, vol. 9, no. 5, pp. 701-730, 2007.

[13] M. del Pino and I. Guerra, "Ground states of a prescribed mean curvature equation," Journal of Differential Equations, vol. 241, no. 1, pp. 112-129, 2007.

[14] N. D. Brubaker and J. A. Pelesko, "Analysis of a one-dimensional prescribed mean curvature equation with singular nonlinearity," Nonlinear Analysis. Theory, Methods \& Applications, vol. 75, no. 13, pp. 5086-5102, 2012.

[15] H. Pan and R. Xing, "Sub- and supersolution methods for prescribed mean curvature equations with Dirichlet boundary conditions," Journal of Differential Equations, vol. 254, no. 3, pp. 1464-1499, 2013.

[16] H. Pan and R. Xing, "A note on the nonexistence of solutions for prescribed mean curvature equations on a ball," Nonlinear Analysis. Theory, Methods \& Applications, vol. 74, no. 18, pp. 7437-7445, 2011.

[17] H. Pan and R. Xing, "Time maps and exact multiplicity results for one-dimensional prescribed mean curvature equations," Nonlinear Analysis. Theory, Methods \& Applications, vol. 74, no. 4, pp. 1234-1260, 2011.

[18] H. Pan and R. Xing, "Exact multiplicity results for a onedimensional prescribed mean curvature problem related to MEMS models," Nonlinear Analysis. Real World Applications, vol. 13, no. 5, pp. 2432-2445, 2012.

[19] H. Pan and R. Xing, "Time maps and exact multiplicity results for one-dimensional prescribed mean curvature equations. II," Nonlinear Analysis. Theory, Methods \& Applications, vol. 74, no. 11, pp. 3751-3768, 2011.
[20] X. Zhang and M. Feng, "Exact number of solutions of a onedimensional prescribed mean curvature equation with concaveconvex nonlinearities," Journal of Mathematical Analysis and Applications, vol. 395, no. 1, pp. 393-402, 2012.

[21] P. Benevieri, J. M. do Ó, and E. S. de Medeiros, "Periodic solutions for nonlinear systems with mean curvature-like operators," Nonlinear Analysis. Theory, Methods \& Applications, vol. 65, no. 7, pp. 1462-1475, 2006.

[22] M. Feng, "Periodic solutions for prescribed mean curvature Liénard equation with a deviating argument," Nonlinear Analysis. Real World Applications, vol. 13, no. 3, pp. 1216-1223, 2012.

[23] J. Cai and B. Lou, "Periodic traveling waves of a mean curvature equation in high dimensional cylinders," Applied Mathematics and Computation, vol. 217, no. 22, pp. 9267-9277, 2011.

[24] P. Benevieri, J. M. do Ó, and E. S. de Medeiros, "Periodic solutions for nonlinear equations with mean curvature-like operators," Applied Mathematics Letters, vol. 20, no. 5, pp. 484492, 2007.

[25] S. Lu, "On the existence of positive periodic solutions for neutral functional differential equation with multiple deviating arguments," Journal of Mathematical Analysis and Applications, vol. 280, no. 2, pp. 321-333, 2003.

[26] R. E. Gaines and J. L. Mawhin, Coincidence Degree, and Nonlinear Differential Equations, vol. 568 of Lecture Notes in Mathematics, Springer, Berlin, Germany, 1977. 


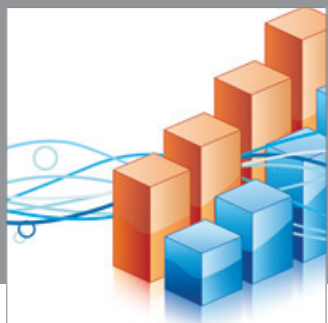

Advances in

Operations Research

mansans

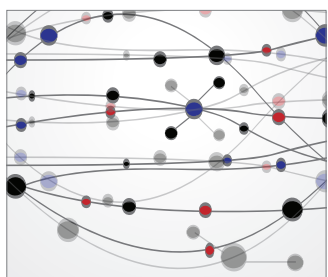

The Scientific World Journal
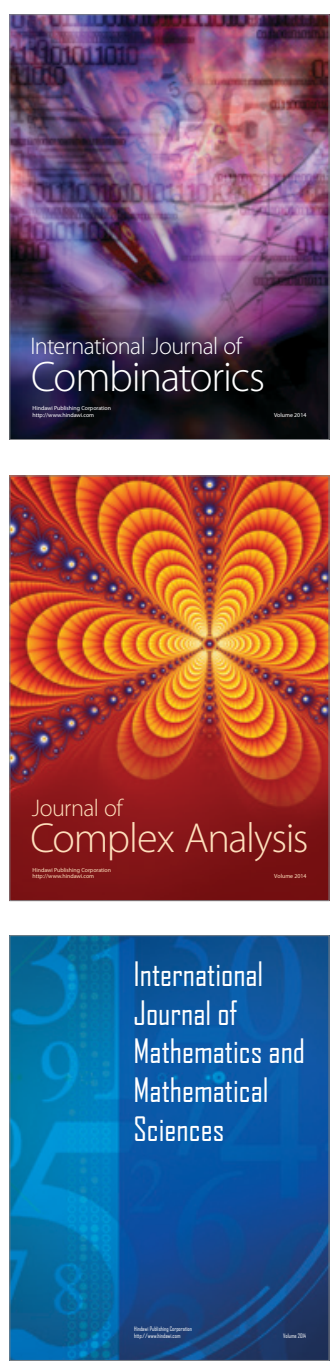
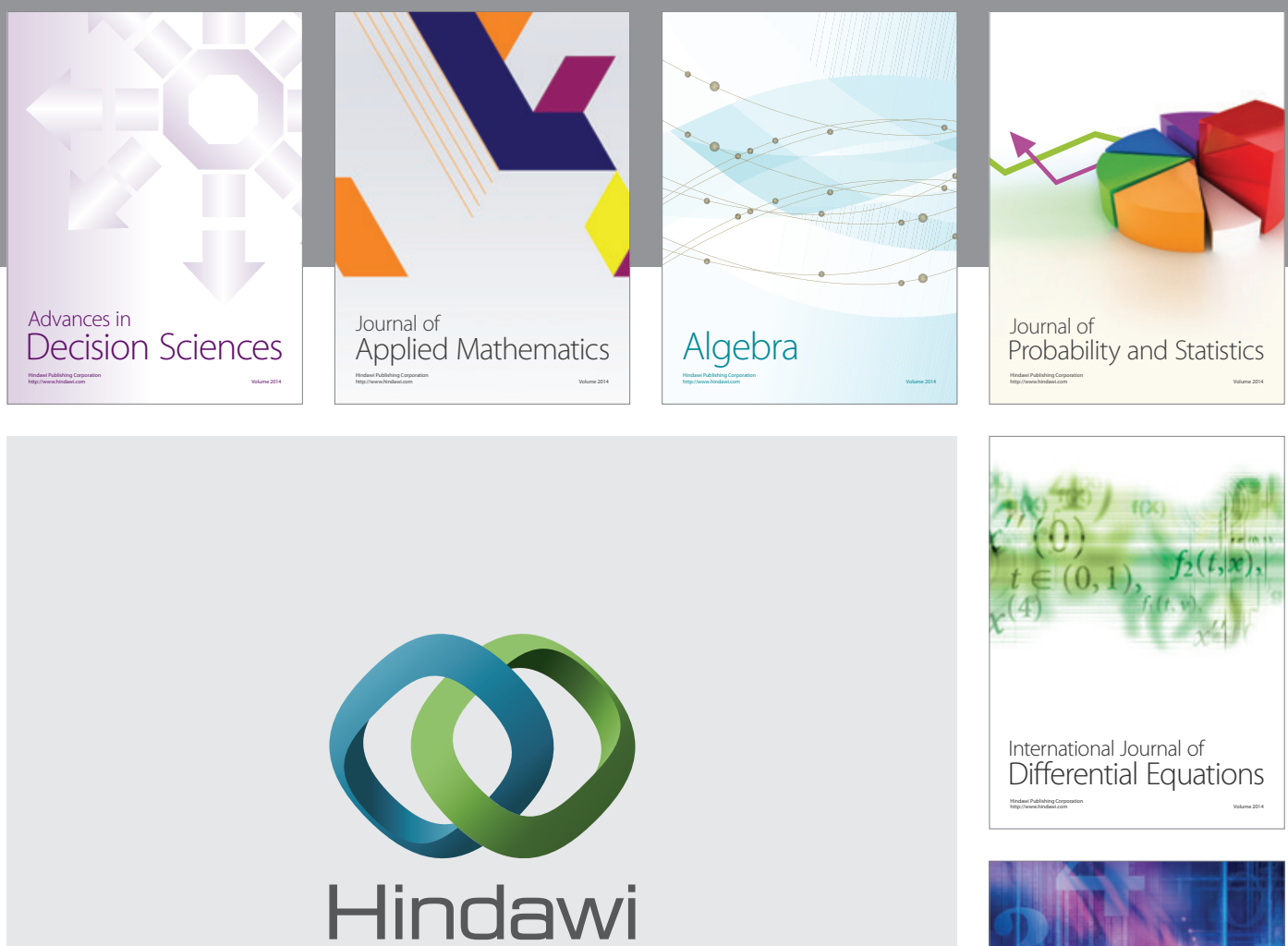

Submit your manuscripts at http://www.hindawi.com
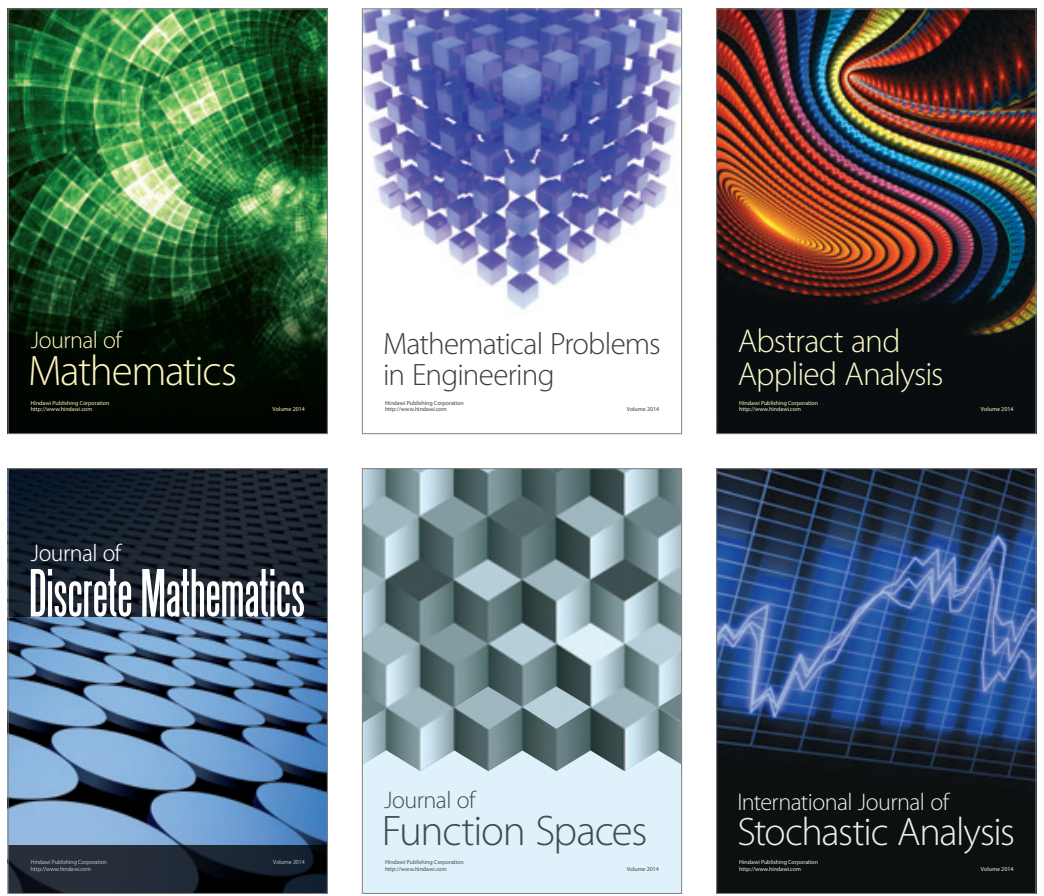

Journal of

Function Spaces

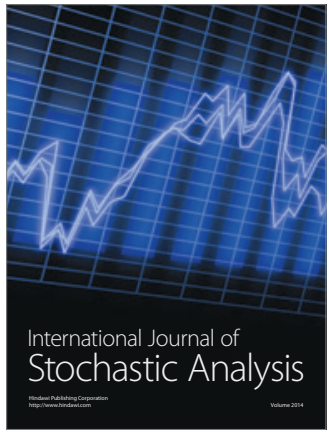

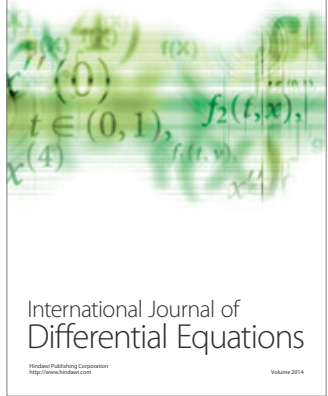
